\title{
Predictors of poor bowel preparations and colonoscopy cancellations in inpatient colonoscopies, a single center retrospective study
}

\author{
Rohit Agrawal $^{1}$, Muhammad Majeed ${ }^{1}$, Bashar M. Attar ${ }^{2}$, Estefania Flores ${ }^{1}$, Zohaib Haque ${ }^{1}$, Sheeba Ba Aqeel ${ }^{1}$, \\ Yuchen Wang ${ }^{2}$, Yazan Abu Omar ${ }^{1}$, Pradeep Parajuli ${ }^{1}$, Melchor Demetria ${ }^{2}$, Seema Gandhi ${ }^{2}$ \\ ${ }^{1}$ Department of Medicine, Cook County Health and Hospital System, Chicago, IL, USA; ${ }^{2}$ Division of Gastroenterology and Hepatology, \\ Department of Medicine, Cook County Health and Hospital System, County, Chicago, IL, USA \\ Contributions: (I) Conception and design: R Agrawal, M Majeed, BM Attar, Y Wang, YA Omar, P Parajuli, M Demetria; (II) Administrative support: \\ None; (III) Provision of study materials or patients: S Ba Aqeel, S Gandhi; (IV) Collection and assembly of data: E Flores, Z Haque; (V) Data analysis \\ and interpretation: R Agrawal, M Majeed, BM Attar, E Flores, Z Haque, S Ba Aqeel, Y Wang, YA Omar, P Parajuli, S Gandhi; (VI) Manuscript \\ writing: All authors; (VII) Final approval of manuscript: All authors. \\ Correspondence to: Rohit Agrawal. Internal Medicine, Cook County Health and Hospital System, 1900 West Polk Avenue, Chicago, IL 60612, USA. \\ Email: rohitagrawal@cookcountyhhs.org.
}

Background: An adequate bowel preparation prior to colonoscopy is a major quality-limiting factor that determines both the diagnostic and therapeutic yield of a colonoscopy. Colonoscopy is considered the gold standard for colon cancer screening and it is the primary approach to the workup of hematochezia, diarrhea and iron deficiency anemia (IDA). Several modifiable factors of bowel prep adequacy have been identified, that account for around $25 \%$ of inadequate bowel preparations in outpatient colonoscopies. However, the literature is sparse when examining the factors associated with inadequate preparations and procedure cancellations in an inpatient hospital setting. We aim to identify factors that affect bowel preparation adequacy and procedure cancellations among diagnostic colonoscopies performed during hospitalization.

Methods: We retrospectively reviewed the electronic medical records of 1,500 consecutive patients who had a diagnostic colonoscopy as an inpatient at a tertiary level hospital over a 2-year period. All patients were administered a clear liquid diet the day prior to the colonoscopy. Patients were then instructed to drink $4 \mathrm{~L}$ of polyethylene glycol (PEG, Golytely) between 5 am to 9 am on the day of the procedure. The clinical course of each case was followed to identify quality of preparations, cancelled procedures and the reasons for cancellations. We applied univariate and multivariate logistic regression analysis to identify variables to predict cancellation and poor preparation.

Results: A total of 1,029 patients were included in the study. 194 (18.8\%) patients had colonoscopy cancellations and 268 (26.0\%) had poor bowel preparations. Multivariate analysis revealed these factors to be associated with colonoscopy cancellations: education at the graduate school level [odds ratio (OR) =1.93, $\mathrm{P}=0.04$, Hispanic ethnicity $(\mathrm{OR}=0.47, \mathrm{P}<0.01)$, hemoglobin level $<10 \mathrm{~g} / \mathrm{dL}(\mathrm{OR}=1.41, \mathrm{P}=0.05)$ and if the colonoscopy was done for other indications $(\mathrm{OR}=0.53, \mathrm{P}=0.04)$. Factors associated with poor bowel preparation on multivariate analysis, were dementia $(\mathrm{OR}=2.44, \mathrm{P}=0.02)$, gastroparesis $(\mathrm{OR}=3.97, \mathrm{P}=0.01)$ and inpatient opioids use $(\mathrm{OR}=1.69, \mathrm{P}=0.04)$.

Conclusions: The rate of colonoscopy cancellations and poor bowel preparations in inpatient colonoscopies were high, and we were able to identify predictors of inadequate colon preparation and procedure cancellations. Exploring more individualized colon preparation regimens based on personal risk factors could reduce the number of inadequate and cancelled colonoscopies in an inpatient setting.

Keywords: Colonoscopy; cancellations; inpatient; inadequate preparation; predictors 
Received: 01 November 2019; Accepted: 10 February 2020; Published: 25 January 2022.

doi: $10.21037 /$ tgh.2020.02.13

View this article at: http://dx.doi.org/10.21037/tgh.2020.02.13

\section{Introduction}

An adequate bowel preparation prior to colonoscopy can greatly affect the diagnostic and therapeutic yield of colonoscopy when performed for colon cancer screening, polyp surveillance or evaluation of gastrointestinal symptoms (1). Adequacy of bowel preparation can limit the diagnostic yield of a colonoscopy and thereby affect adenoma detection rate (ADR), cecal intubation rate, withdrawal and procedural time and the interval between repeat procedures (1-4).

The quality of bowel preparation can be affected by many patient factors, including poor sociodemographic status, presence of comorbidities, certain medications and the timing of colonoscopy. These factors account for approximately $25 \%$ of inadequate bowel preparations $(1,2,5,6)$. With approximately 11 million colonoscopies performed just in 2013 we can acknowledge the significant healthcare and economic burden of inadequate preparations, which can lead to missed colon cancers, repeat procedures and potentially prolonged hospitalizations (7). Optimizing inpatient bowel preparation has significant ramifications as utilization of inpatient care is an important focus in hospital medicine, as it can prevent repeated procedures as an inpatient. Just recently, Medicare and Medicaid emphasized offering more care in the ambulatory setting so as to reduce the cost of medical care (8). Likewise, there is a focus on reducing the length of stay (LOS) for these patients, as LOS directly affects the cost of hospitalization. Having an inadequate bowel prep usually means there will be a 2-day greater LOS and over $\$ 8,000$ more in hospitalization costs (9).

Colonoscopies performed on an inpatient population vary in terms of patient demographics, medical complexity and indications for procedure. Colonoscopies performed as an inpatient have been found to have higher rates of poor bowel preparations, as compared to outpatient procedures, with some studies noting it to be as high as $50 \%(10-12)$. The same risk factors for a poor bowel preparation in an outpatient colonoscopy are also noted to be independent risk factors for poor inpatient bowel preparations; which result in higher number of repeat procedures and increased length of hospital stay and costs $(3,9)$.

There is very little literature looking at inpatient colonoscopy prep quality and resultant cancellations.
There is a need to identify factors associated with inadequate preparations in order to develop models that help improve the quality of inpatient bowel preparations. We aim to identify factors that are associated with poor bowel preparation and procedure cancellations in inpatient colonoscopies.

\section{Methods}

\section{Database setup}

We retrospectively reviewed the electronic medical records of 1,500 consecutive patients who underwent an inpatient colonoscopy between January 2014 and January 2016, for any reason during hospitalization at a tertiary level hospital. We excluded patients $<18$ years of age, pregnant females, and those who had missing data as outlined in the variables section.

The present study was approved by the Institutional Review Board of the Cook County Health \& Hospitals System, Chicago, IL. The database was set up and maintained by the Department of Medicine, Cook County Health \& Hospitals System. The study was conducted in accordance with the Declaration of Helsinki (as revised in 2013). Written consent from patients to publish this study was waived.

\section{Variables}

The variables extracted for the review were age, gender, body mass index (BMI), diet preference, ethnicity, education level, medical history and alcohol, tobacco, and illicit substance use. Data regarding medications such as use of calcium channel blockers, TCA, iron supplements and inpatient opioid use were collected. We obtained biochemical data such as hemoglobin ( $\mathrm{Hb})$, glycated hemoglobin (HbA1C), serum calcium, serum albumin and thyroid stimulating hormone (TSH) at the time of colonoscopy. The indication for the colonoscopy and whether the inpatient gastroenterology consult service was on formal consultation was also collected.

The clinical course of each case was followed with timing of colonoscopy, cancellation of colonoscopy and the reasons for cancellation. The quality of preparations was 
collected by reviewing the colonoscopy report. Patients with incomplete data were excluded from the study.

\section{Bowel preparation protocol}

All patients were administered a clear liquid diet the day prior to the colonoscopy. Patients were instructed to drink 4 liters of polyethylene glycol (PEG, Golytely) between 5 am and 9 am on the day of the procedure. The nurse in charge was responsible for the completion of the bowel preparation also ensuring the patient had clear fluid like stools prior to the colonoscopy. The procedure was cancelled if the quality of stools post bowel preparation was inadequate. For patients who were rescheduled for the procedure, the entire bowel preparation with polyethylene glycol was repeated with nurses ensuring the completion of preparation and an adequate quality of stools.

\section{Quality of bowel preparation}

Quality of bowel preparations were reported as good/ adequate, fair or poor. Good or adequate bowel preparation was defined as absent or minimal stool with some amount of clear fluid requiring suctioning. Fair preparation was defined as the presence of semisolid debris requiring more aggressive suctioning and poor preparation was presence of solid or semisolid debris that could not be cleared even with aggressive suctioning (10).

\section{Statistical analysis}

We performed analyses to describe and summarize the distributions of variables. We use the Student's $t$-test, Wilcoxon rank sum test, or Kruskal-Wallis test to compare continuous nonparametric variables, and the Chi-square test or Fisher's exact test to compare categorical variables. We applied univariate and multivariate logistic regression analysis on the relevant demographic and biochemical variables to predict cancellation and poor preparation. All statistical analyses were performed using STATA (Version 14.0, College Station, TX). Otherwise, we considered P values of $<0.05$ to be statistically significant.

\section{Results}

\section{Cobort characteristics}

A total of 1,029 patients were included in the study with a mean age of 55.6 (SD 14.1) years (Table 1). Males comprised $64.4 \%$ of the sample size and majority were African American in ethnicity (55.0\%). Diabetes mellitus was the most prevalent co-morbid condition (28.5\%), followed by malignancy (23.5\%), psychiatric disorder $(16.1 \%)$, stroke $(4.7 \%)$, and dementia $(3.1 \%)$. The most common indications for a colonoscopy were gastrointestinal bleeding/iron deficiency anemia $(67.5 \%)$, constipation, diarrhea or abdominal pain $(11.8 \%)$ and evaluation of malignancy $(7.2 \%)$, while (13.3\%) patients had colonoscopy for "other reasons".

\section{Factors resulting in poor bowel preparation}

Patients in our cohort were divided into 2 groups, good/ adequate $v s$. poor bowel preparation. In the univariate analysis, dementia $(\mathrm{P}=0.02)$, gastroparesis $(\mathrm{P}<0.01)$, constipation $(\mathrm{P}=0.04)$, taking TCA's $(\mathrm{P}=0.04)$ and receiving opioids inpatient $(\mathrm{P}<0.01)$ were associated with poor bowel preparation (Table 2). Factors associated with poor bowel preparation were further analyzed using a multivariate analysis, we found that dementia ( $\mathrm{OR}=2.44, \mathrm{P}=0.02)$, gastroparesis $(\mathrm{OR}=3.97, \mathrm{P}=0.01)$ and taking opioids as an inpatient $(\mathrm{OR}=1.69, \mathrm{P}<0.01)$ were associated with poor bowel preparation.

\section{Factors resulting in colonoscopy cancellation}

A total of 194 (18.8\%) patients had colonoscopy cancellations. Fifty patients refused procedure, 52 patients failed to complete the preparation, 41 patients had scheduling issues and 51 patients had their procedure cancelled due to medical conditions such as uncontrolled hypertension, atrial fibrillation or flutter, sepsis, electrolyte abnormalities, thrombocytopenia, or unspecified conditions. In the univariate analysis, educational at graduation level ( $\mathrm{P}=0.03)$, ethnicity $(\mathrm{P}<0.01)$, constipation $(\mathrm{P}=0.02)$, taking iron supplements $(\mathrm{P}=0.02)$, Hb level $<10 \mathrm{~g} / \mathrm{dL}(\mathrm{P}<0.01)$ and if the colonoscopy was due to other indications $(\mathrm{P}<0.01)$ were associated with colonoscopy cancellations (Table 3). "Other indications" included a diverse group mainly consisting of IBD surveillance and endoscopic work up due to abnormal abdominal imaging findings. In the multivariate analysis, education level at graduation school $(\mathrm{OR}=1.93, \mathrm{P}=0.04$, Hispanic ethnicity $(\mathrm{OR}=0.47, \mathrm{P}<0.01)$, other ethnicity (OR $=0.01, \mathrm{P}=0.02)$, Hb level $<10 \mathrm{~g} / \mathrm{dL}$ (OR $=1.41, \mathrm{P}=0.05)$ and if the colonoscopy was done for other indications $(\mathrm{OR}=0.53, \mathrm{P}=0.04)$ were associated with higher 
Table 1 Baseline characteristics of study population

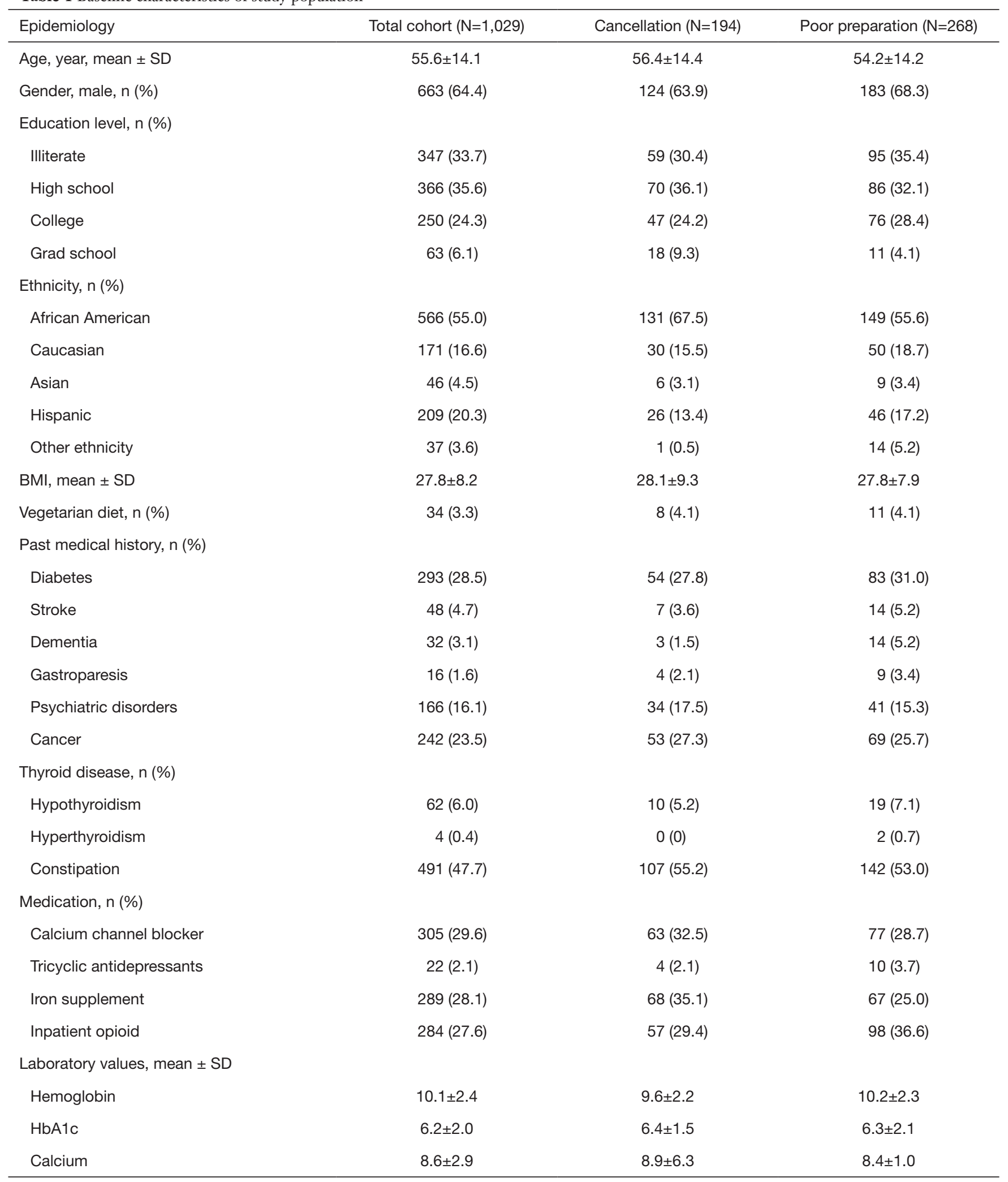

Table 1 (continued) 
Table 1 (continued)

\begin{tabular}{lccc}
\hline Epidemiology & Total cohort $(\mathrm{N}=1,029)$ & Cancellation $(\mathrm{N}=194)$ & Poor preparation $(\mathrm{N}=268)$ \\
\hline Indication for colonoscopy, $\mathrm{n}(\%)$ & & & \\
Bleeding/iron deficiency anemia & $695(67.5)$ & $145(74.7)$ & $186(69.4)$ \\
Malignancy evaluations & $74(7.2)$ & $23(11.9)$ & $20(7.5)$ \\
Constipation/diarrhea/pain & $121(11.8)$ & $14(7.2)$ & $26(9.7)$ \\
Other indications & $137(13.3)$ & $43(22.2)$ & $36(13.4)$ \\
Prior colonoscopy, $\mathrm{n}(\%)$ & $246(23.9)$ & $78(40.2)$ & $59(22.0)$ \\
Gl consult, $\mathrm{n}(\%)$ & $473(46.0)$ & $142(53.0)$ & \\
\hline
\end{tabular}

$\mathrm{SD}$, standard deviation; HbA1c, glycated hemoglobin; Gl, gastroenterology.

Table 2 Univariate and multivariate analysis of factors associated with inadequate bowel preparations

\begin{tabular}{|c|c|c|c|c|}
\hline Characteristics & \multicolumn{2}{|c|}{ Univariate unadjusted } & \multicolumn{2}{|c|}{ Multivariate adjusted } \\
\hline Elderly (age >65 years) & 0.71 & 0.07 & 0.71 & 0.08 \\
\hline Male & 126 & 0.13 & & \\
\hline \multicolumn{5}{|l|}{ Education level } \\
\hline High school & 0.81 & 0.23 & 0.85 & 0.36 \\
\hline College & 1.15 & 0.42 & 1.20 & 0.33 \\
\hline Grad school & 0.56 & 0.10 & 0.59 & 0.14 \\
\hline Dementia & 2.27 & 0.02 & 2.44 & 0.02 \\
\hline Tricyclic acid & 2.42 & 0.04 & 2.24 & 0.07 \\
\hline Iron supplement & 0.81 & 0.19 & & \\
\hline Inpatient opioid & 1.78 & $<0.01$ & 1.69 & $<0.01$ \\
\hline
\end{tabular}

odds of colonoscopy cancellations.

\section{Repeat colonoscopies}

Fifty-four (27.8\%) patients with colonoscopy cancellations had repeat colonoscopies within an average of 1.9 days from cancellation. Despite repeat bowel preparations, only $18.5 \%$ of these patients' colonoscopies achieved adequate preparation on their repeat colonoscopy. The percentage of repeat colonoscopies was $17.9 \%$ in patients with poor bowel preparations. Around $31.3 \%$ of these patients had adequate bowel preparations in repeat procedures. The average time between repeat colonoscopies in this cohort of patients was 1.7 days (Table 4).

\section{Discussion}

Our analysis revealed a $26 \%(\mathrm{n}=268)$ rate of inadequate bowel preparations for inpatient colonoscopies, which is consistent with other studies that report rates ranging from $18 \%$ to $35 \%$ (12). Prolonged immobility, narcotic use and multiple comorbidities are common in an inpatient population and are associated with higher rates of poor bowel preparations (13-15). Opioid usage was identified as 
Table 3 Univariate and multivariate analysis of factors associated with colonoscopy cancellations

\begin{tabular}{|c|c|c|c|c|}
\hline Characteristics & \multicolumn{2}{|c|}{ Univariate unadjusted } & \multicolumn{2}{|c|}{ Multivariate adjusted } \\
\hline \multicolumn{5}{|l|}{ Education level } \\
\hline Illiterate & Ref & N/A & Ref & N/A \\
\hline High school & 1.15 & 0.46 & 1.09 & 0.68 \\
\hline Grad school & 1.95 & 0.03 & 1.93 & 0.04 \\
\hline \multicolumn{5}{|l|}{ Ethnicity } \\
\hline African American & Ref & N/A & Ref & N/A \\
\hline Caucasian & 0.7 & 0.12 & 0.75 & 0.2 \\
\hline Other ethnicity & 0.09 & 0.02 & 0.01 & 0.02 \\
\hline Dementia & 0.44 & 0.18 & & \\
\hline Cancer & 1.28 & 0.16 & & \\
\hline Constipation & 1.44 & 0.02 & 1.29 & 0.13 \\
\hline Iron supplement & 1.49 & 0.02 & 1.26 & 0.19 \\
\hline Hemoglobin $<10$ & 1.64 & $<0.01$ & 1.41 & 0.05 \\
\hline \multicolumn{5}{|l|}{ Indication for colonoscopy } \\
\hline Bleeding/iron deficiency anemia & Ref & N/A & Ref & $\mathrm{N} / \mathrm{A}$ \\
\hline
\end{tabular}

Table 4 Bowel preparation characteristics in patients with repeat colonoscopies

\begin{tabular}{lcc}
\hline Characteristics & Cancellation $(\mathrm{N}=194)$ & Poor preparation $(\mathrm{N}=268)$ \\
\hline Repeat procedure, $\mathrm{n}(\%)$ & $54(27.8)$ & $48(17.9)$ \\
Preparation, $\mathrm{n}(\%)$ & & $15(31.3)$ \\
Adequate & $10(18.5)$ & $20(41.7)$ \\
Fair & $30(55.6)$ & $13(27.1)$ \\
Poor & $14(25.9)$ & $1.7(1.3)$ \\
\hline
\end{tabular}

a factor associated with poor preparations in our analysis. The link between opiate use causing delayed colon transit and constipation is well established. Over $80 \%$ of patients complain of constipation due to side effects of opiates, which is likely responsible for inadequate bowel preparation, failed colonoscopies and hence the need for repeated procedures $(16,17)$. Patients with dementia were more likely to have poor bowel preparations in our analysis. 
This could be secondary to difficulties in swallowing, altered gastrointestinal motility, problems with mobility, and also the comprehension and adherence to the preparation (18).

Graduate level education, Hispanic ethnicity, anemia with a hemoglobin level $<10 \mathrm{gm} / \mathrm{dL}$ and "other indications" for colonoscopy were associated with colonoscopy cancellations. While there is existing literature describing factors that predict a poor bowel prep, there is only limited literature looking at all factors leading to cancellation of colonoscopy performed as an inpatient. A handful of studies have identified screening colonoscopies and sociodemographic factors such as young age, female sex and type of insurance to be associated with colonoscopy cancellations $(19,20)$. There is evidence associating anemia with higher prevalence of atrial fibrillation and uncontrolled hypertension which could explain partially why there were higher rates of colonoscopy cancellations in anemic patients $(21,22)$. It is unclear why other factors we identified are associated with colonoscopy cancellations.

In a meta-analysis of 24 studies, inpatient status, diabetes mellitus, narcotic use, constipation, stroke, and TCA use among other factors, were associated with inadequate bowel preparation (14). Gandhi et al. identified similar risk factors to be significant predictors of poor preparations in a meta-analysis of 67 studies that included 75,818 patients. Similarly, our analysis demonstrates significant associations of poor bowel preparation and several of the other reasons mentioned above (5).

In a study of 12,787 inpatient and outpatient colonoscopies where majority were outpatient procedures, the percentage of suboptimal preparation was $24 \%$ and among these, repeat examinations were performed in $17 \%$ within 3 years (23). Our study showed a similar percentage (17.9\%) of repeated colonoscopy in patients with poor preparations, however the average time between repeat colonoscopies was 1.7 days. The rate of repeat colonoscopies was slightly higher in those who had cancellations with average time to repeat procedure being 1.9 days. Inadequate bowel preparations have been associated with increased length of hospital stay and costs. Although not formally studied in our analysis, poor preparations and cancellations added at least 2 days to patients undergoing repeat procedures which is an additional cost for patients and hospitals (9).

This study has several limitations. This was a retrospective study where causation cannot be established, the evaluation of the quality of bowel preparation was not standardized, and we did not specifically assess compliance. These factors could lead to measurement bias and overestimation of poor preparation quality.

\section{Conclusions}

All these findings support the identification of predictors of inadequate colon preparation, and further emphasize the need to explore individualized colon preparation and to apply efforts focused on modification of inpatient medication. Acknowledging theses factor to optimize bowel preparation can alleviate the burden of repeat colonoscopies and the increased cost to healthcare system. Future prospective, randomized controlled trials are needed to establish individualized approaches of bowel preparations for patients with above identified risk factors.

\section{Acknowledgments}

Funding: None.

\section{Footnote}

Conflicts of Interest: The authors have no conflicts of interest to declare.

Ethical Statement: The authors are accountable for all aspects of the work in ensuring that questions related to the accuracy or integrity of any part of the work are appropriately investigated and resolved. The present study was approved by the Institutional Review Board of the Cook County Health \& Hospitals System, Chicago, IL. The study was conducted in accordance with the Declaration of Helsinki (as revised in 2013). Written consent from patients to publish this study was waived.

Open Access Statement: This is an Open Access article distributed in accordance with the Creative Commons Attribution-NonCommercial-NoDerivs 4.0 International License (CC BY-NC-ND 4.0), which permits the noncommercial replication and distribution of the article with the strict proviso that no changes or edits are made and the original work is properly cited (including links to both the formal publication through the relevant DOI and the license). See: https://creativecommons.org/licenses/by-nc$\mathrm{nd} / 4.0 \%$.

\section{References}

1. Johnson DA, Barkun AN, Cohen LB, et al. US Multi- 
Society Task Force on Colorectal Cancer. Optimizing adequacy of bowel cleansing for colonoscopy: recommendations from the US multi-society task force on colorectal cancer. Gastroenterology 2014;147:903-24.

2. Harewood GC, Sharma VK, de Garmo P. Impact of colonoscopy preparation quality on detection of suspected colonic neoplasia. Gastrointest Endosc 2003;58:76-9.

3. Rex DK, Imperiale TF, Latinovich DR, et al. Impact of bowel preparation on efficiency and cost of colonoscopy. Am J Gastroenterol 2002;97:1696-700.

4. Senore C, Ederle A, Fantin A, et al. Acceptability and sideeffects of colonoscopy and sigmoidoscopy in a screening setting. J Med Screen 2011;18:128-34.

5. Gandhi K, Tofani C, Sokach C, et al. Patient Characteristics Associated with Quality of Colonoscopy Preparation: A Systematic Review and Meta-analysis. Clin Gastroenterol Hepatol 2018;16:357-369.e10.

6. Strate LL, Syngal S. Timing of colonoscopy: impact on length of hospital stay in patients with acute lower intestinal bleeding. Am J Gastroenterol 2003;98:317-22.

7. Peery AF, Crockett SD, Murphy CC, et al. Burden and Cost of Gastrointestinal, Liver, and Pancreatic Diseases in the United States: Update 2018. Gastroenterology 2019;156:254-272.e11.

8. Eddy DM, Shah R. A simulation shows limited savings from meeting quality targets under the Medicare Shared Savings Program. Health Aff (Millwood) 2012;31:2554-62.

9. Yadlapati R, Johnston ER, Gregory DL, et al. Predictors of Inadequate Inpatient Colonoscopy Preparation and Its Association with Hospital Length of Stay and Costs. Dig Dis Sci 2015;60:3482-90.

10. Chorev N, Chadad B, Segal N, et al. Preparation for colonoscopy in hospitalized patients. Dig Dis Sci 2007;52:835-9.

11. Hendry PO, Jenkins JT, Diament RH. The impact of poor bowel preparation on colonoscopy: a prospective single centre study of 10,571 colonoscopies. Colorectal Dis 2007;9:745-8.

12. Ness RM, Manam R, Hoen H, et al. Predictors of inadequate bowel preparation for colonoscopy. Am J Gastroenterol 2001;96:1797-802.

13. Froehlich F, Wietlisbach V, Gonvers JJ, et al. Impact of colonic cleansing on quality and diagnostic yield of colonoscopy: the European Panel of Appropriateness of Gastrointestinal Endoscopy European multicenter study. Gastrointest Endosc 2005;61:378-84.
14. Mahmood S, Farooqui SM, Madhoun MF. Predictors of inadequate bowel preparation for colonoscopy: a systematic review and meta-analysis. Eur J Gastroenterol Hepatol 2018;30:819-26.

15. Qureshi A, Ismail S, Azmi A, et al. Poor bowel preparation in patients undergoing colonoscopy. Med J Malaysia 2000;55:246-8.

16. Leppert $W$. The impact of opioid analgesics on the gastrointestinal tract function and the current management possibilities. Contemp Oncol (Pozn) 2012;16:125-31.

17. Kushnir VM, Bhat P, Chokshi RV, et al. The impact of opiate pain medications and psychoactive drugs on the quality of colon preparation in outpatient colonoscopy. Dig Liver Dis 2014;46:56-61.

18. Yee R, Manoharan S, Hall C, et al. Optimizing bowel preparation for colonoscopy: what are the predictors of an inadequate preparation? Am J Surg 2015;209:787-92; discussion 792 .

19. Greenspan M, Chehl N, Shawron K, et al. Patient Nonadherence and Cancellations Are Higher for Screening Colonoscopy Compared with Surveillance Colonoscopy. Dig Dis Sci 2015;60:2930-6.

20. Denberg TD, Melhado TV, Coombes JM, et al. Predictors of nonadherence to screening colonoscopy. J Gen Intern Med 2005;20:989-95.

21. Keskin M, Ural D, Altay S, et al. Iron deficiency and hematinic deficiencies in atrial fibrillation: A new insight into comorbidities. Turk Kardiyol Dern Ars 2018;46:103-10.

22. Paul B, Wilfred NC, Woodman R, et al. Prevalence and correlates of anemia in essential hypertension. Clin Exp Pharmacol Physiol 2008;35:1461-4.

23. Lebwohl B, Kastrinos F, Glick M, et al. The impact of suboptimal bowel preparation on adenoma miss rates and the factors associated with early repeat colonoscopy. Gastrointest Endosc 2011;73:1207-14.

doi: $10.21037 /$ tgh.2020.02.13

Cite this article as: Agrawal R, Majeed M, Attar BM, Flores E, Haque Z, Ba Aqeel S, Wang Y, Omar YA, Parajuli P, Demetria M, Gandhi S. Predictors of poor bowel preparations and colonoscopy cancellations in inpatient colonoscopies, a single center retrospective study. Transl Gastroenterol Hepatol 2022;7:4. 